Bazyli Degórski, O.S.P.P.E.

Pontificia Università S. Tommaso d'Aquino, Roma

DOI: http://dx.doi.org/10.12775/BPTh.2013.010

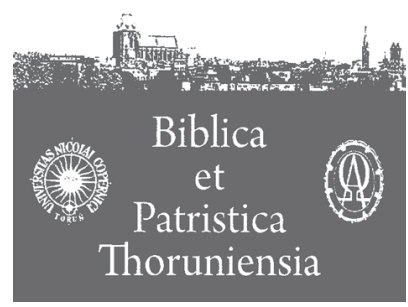

$6(2013) \quad$ ISSN $1689-5150$

\title{
Il Libro di Giobbe nella letteratura monastica delle origini (secoli IV-V)
}

\section{The Book of Job is the oldest monastic literature of the $4^{\text {th }}$ and $5^{\text {th }}$ century}

\section{Księga Hioba w najstarszym piśmiennictwie monastycznym (IV-V wiek)}

Słowa klucze: literatura monastyczna; Księga Hioba.

Key words: monastic literature; Job; patristic interpretation.

Streszczenie. Wiek IV i V to najważniejszy okres dla rodzącego się monastycyzmu. Niniejszy artykuł ukazuje, w jakim stopniu piśmiennictwo monastyczne tego okresu odwoływało się do Księgi Hioba. W artykule uwzględniono wszystkich znanych dzisiaj trzydziestu ośmiu pisarzy monastycznych tego okresu, analizując ich sto osiem dzieł. Zajęliśmy się jednakże tylko tymi, które przeznaczone zostały dla mnichów, nie badaliśmy natomiast pism skierowanych do innych odbiorców. Chociaż nasze badania rozciągnęliśmy także na autorów łacińskich, najwięcej materiału znaleźliśmy u Ojców wschodnich. Jest to zrozumiałe, gdyż niemalże wszyscy pisarze monastyczni na Zachodzie byli ukształtowani przez monastycyzm wschodni, który przecież narodził się na Wschodzie. $Z$ tego też względu monastyczne dziedzictwo Wschodu jest o wiele bogatsze niż Zachodu.

Na początku artykułu ukazaliśmy, w jaki sposób pisarze monastyczni IV i V wieku korzystali z Księgi Hioba. W drugiej części zajęliśmy się pokrótce wpływem Księgi Hioba na duchowość rodzącego się monastycyzmu. Obie części pozwoliły na sformułowanie ogólniejszych wniosków. W piśmiennictwie rodzącego się monastycyzmu znajdujemy aż 153 odniesienia do Księgi Hioba, z czego 91 to dosłowne cytaty. Taki stan rzeczy umieszcza to natchnione pismo na drugim miejscu wśród wszystkich ksiąg mądrościowych Biblii (zaraz po Księdze Przysłów, która została wykorzystana 412 razy, z czego 314 ad litteram). Pisarze monastyczni IV-V wieku najczęściej korzystali z następujących fragmentów Księgi Hioba: 1,21 (12 razy), 2,7 (6 razy), 40,11 (sześć razy), 40,24 (pięć razy).

Księga Hioba zawdzięcza niewątpliwie swe powodzenie w piśmiennictwie monastycznym pierwszych wieków surowemu sposobowi życia swego bohatera. Był to dla pierwszych mnichów wspaniały wzór do naśladowania, a zwycięstwo końcowe Hioba 
napełniało ich optymizmem i nadzieją, iż wszystkie wyrzeczenia, cierpienia i pokuty zostaną nagrodzone przez Boga.

Abstract. The article reveals taking advantage from the book of Job by using the oldest monastic written text (IV and V century) - the most important period the beginnings of the western and eastern monasticism. The article has examined all the authors from both the $4^{\text {th }}$ and $5^{\text {th }}$ century without exception: thirty eight writers analysing their one hundred eight works. We took special attention to the works written for monastic orders and did not examine works for other recipients.

It is obvious that our analysis has extended to authors from the west (Latin) - majority of materials we found with the monks from the eastern tradition and by two reasons: firstly the authors from the west were educated in the eastern tradition and secondly the eastern monastic inheritance from that time was more richer then the west's. In the beginning of the article the book of Job is used by actual monastic writers from the $4^{\text {th }}$ and $5^{\text {th }}$ century. In the second part we addressed the influence of the book of Job mentally and the ascetic beginnings of monasticism. On the other hand towards the end part of the article has been summarised. In literary references from the beginnings of monasticism the book of Job is in second place amongst all the books of Wisdom in the Bible (straight after the book of Proverbs which is in first place.). The book of Job is used 153 times of which 91 are to the letter.

Job undoubtedly owes his success in monastic writings from the $1^{\text {st }}$ century from his own austere way of life, which gave the first monks a splendid example to replicate, but Job's final victory enlightened monks with hope and optimism with their self abnegation, suffering and penance will be rewarded by God. Monastic writers of the $4^{\text {th }}$ and $5^{\text {th }}$ century made use off especially the following fragments of the book of Job: 1,21 (12 times), 2,7 (6 times), 40,11 (six times), 40,24 (five times).

\section{Introduzione}

Tl presente articolo prende in esame l'utilizzazione del Libro di Giobbe nella più antica letteratura monastica, ossia dei secoli IV e V, il periodo più significativo del monachesimo nascente, sia orientale che occidentale.

Per stilare l'articolo, abbiamo preso in esame tutti gli autori monastici del periodo in questione, senza esclusione di provenienza o di fama; trentotto autori, di cui abbiamo analizzato centocinque opere ${ }^{1}$. Si tratta soltanto delle opere

1 Non si riscontra alcuna utilizzazione dei libri sapienziali come tali e, quindi, del Libro di Giobbe nelle seguenti opere monastiche: Basilius Caesariensis, Moralia, in PG [= J.-P. Migne (ed.), Patrologiae cursus completus. Series Graeca, Parisiis 1857-1866] 31, coll. 700-869; Idem, Praevia institutio ascetica, in PG 31, coll. 620-625; Iohannes Chrysostomus, Comparatio regis et monachi, in PG 47, coll. 387-392; Diadochus, Visio, in SCh [= Sources Chrétiennes, Paris 1941 ss.] 5 bis, pp. 169-179; Eutropius, Epistula de condemnanda haere- 
scritte esclusivamente per i monaci; non abbiamo, quindi, preso in considerazione scritti diretti ad altri destinatari ${ }^{1}$. Va da sé che, nonostante l'ampliamento anche agli autori occidentali, sono i Padri orientali a fare la parte del leone, per così dire, e questo per due motivi: innanzitutto perché alcuni autori occidentali furono ugualmente formati alla scuola monastica orientale e, quindi, ne conservano i tratti; in secondo luogo perché il patrimonio monastico orientale di questo periodo è molto superiore a quello occidentale, tanto da fagocitarlo non solo nella quantità, ma anche nella rilevanza di cui godé in seno alla tradizione monastica in genere.

Dell'analisi dettagliata vengono offerte solo le considerazioni che se ne sono desunte, organizzate in due tempi distinti: in un primo momento, si fisserà l'attenzione sulla presenza quantitativa e qualitativa del Libro di Giobbe nella produzione spirituale monastica del IV e del V secolo; in un secondo tempo, si considererà la valenza esegetica del suo impiego. Infine, saranno dati dei rilievi conclusivi.

ditate, in PL 30, coll. 47-52; Evagrius Ponticus, Capitula XXXIII, in PG 40, coll. 1264-1268; Idem, Spiritales sententiae, in PG 40, coll. 1268-1269; Idem, De octo vitiosis cogitationibus ad Anatolium, in PG 40, coll. 1272-1278; Idem, Sententiae, in PG 40, coll. 1277-1286; Idem, Institutio ad monachos, in PG 79, coll. 1235-1240; Idem, Capita paraenetica, in PG 79, coll. 1249-1264; Hieronymus, Contra Helvidium, in PL [= J.-P. Migne (ed.), Patrologiae cursus completus. Series Latina, Parisiis 1841-1864] 23, coll. 193-216; Lupus Trecensis, Vita S. Lupi, in MGH [= Monumenta Germaniae Historica, Hannover - Berlin 1826 ss.] scr. mer. 7, coll. 295-302; Marcus Eremita, Ad Nicolaum praecepta animae salutaria, in PG 65, coll. 1027-1054; Idem, De baptismo, in PG 65, coll. 985-1028; Nilus, In Albianum oratio, in PG 79, coll. 696-712; Idem, De magistris et discipulis, in P. van den Ven (ed.), Un opuscule inédit attribué à S. Nil, in Mélange G. Kurth, II, Liège 1908, pp. 73-81; Nilus, Ad Eucarpium monachum, in PG 89, col. 357 (perduto; resta solo un frammento); Salvianus, Epistulae I, II, VIII, in SCh 176, pp. 76-132; Hilarius Arelatensis, Epistula ad Eucherium Lugdunensem, in CSEL [= Corpus Scriptorum Ecclesiasticorum Latinorum, Vindobonae 1865 ss.] 31; Sidonius Apollinaris, Epistulae ad Lupum, in PL 58, coll. 551, 554, 558, 626; Sulpitius Severus, Dialogi, in CSEL 1, pp. 152-216; Theodorus Orsiesii coadiutor, Epistula, in PL 23, coll. 104-106; Theophilus Alexandrinus, Epistula de monacho Theodoro, in CSEL 55, pp. 142-143.

1 Non sono state prese in considerazione le opere che, pur trattando di argomenti spirituali o ascetici, non erano indirizzate agli ambienti monastici propriamente detti (ad esempio: la letteratura sulla verginità, i cui destinatari non sono monaci o monache). Non compaiono, altresì, gli scritti nei quali non si trova alcun riferimento al Libro di Giobbe, che, in ogni modo, sono stati consultati. 


\section{Presenza del Libro di Giobbe nella letteratura monastica delle origini}

\subsection{Singoli autori monastici}

Un primo dato da registrare è un'alta frequenza con la quale il Libro di Giobbe viene utilizzato nella letteratura monastica del IV e del V secolo.

Sant'Anfilochio di Iconio, nel Contra gli apotactiti e i gemelliti, cita letteralmente il Libro di Giobbe soltanto una sola volta ${ }^{2}$.

Sant'Antonio Abate, nelle Epistulae, adopera il Libro di Giobbe ben tre volte, di cui due volte letteralmente ${ }^{3}$. Nei Sermones XX ad filios suos monachos, egli si serve del Libro di Giobbe una sola volta, usandolo non letteralmente ${ }^{4}$.

Gli Apophthegmata Patrum si servono del Libro di Giobbe cinque volte, presentandone tre citazioni dirette ${ }^{5}$.

Nella Vita S. Antonii, sant'Atanasio di Alessandria utilizza il Libro di Giobbe sette volte, di cui solo due citazioni sono dirette ${ }^{6}$.

San Basilio di Cesarea, nel De baptismo, citta due volte letteralmente il Libro di Giobbe ${ }^{7}$. Nel De iudicio Dei, si appella una sola volta a Giobbe, non citandolo esplicitamente ${ }^{8}$. Nelle Regulae brevius tractatae, egli fa riferimento a Giobbe tre

2 Cf. Gb ${ }^{\star} 15,16$ = Apot. et gem., in G. Ficker (ed.), Amphilochiana, I, Leipzig 1906, p. 52. Nota bene: l'asterisco precedente il riferimento scritturistico contrassegna le citazioni esplicite.

3 Cf. Gb ${ }^{\star} 10,6-7 ; \mathrm{Gb}{ }^{\star 39}, 34-35=$ Epistula 16, in PG 40, col. 1035 BC; Gb 40, $11=$ Epistula 17, in PG 40, col. 1044 B.

4 Cf. Gb 40, 11 = Sermones XX ad filios suos monachos 20, in PG 40, col. 976.

5 Cf. Gb ${ }^{\star} 1,21$ = Apophthegmata Patrum. Macarius Aegyptius 18, in PG 65, col. 269; Gb 1, 22 = Apophthegmata Patrum. Iohannes Curtus 45, in PG 65, col. 204; Gb *15, $15=$ Apophthegmata Patrum. Zeno 4, in PG 65, col. 176; Gb 34, 21 = Apophthegmata Patrum. Gelasius 6, in PG 65, col. 153; Gb *40, 5 = Apophthegmata Patrum. Evagrius 7, in PG 65, col. 176.

6 Cf. Gb 1, 12 ss., Gb 2, 7 = Vita S. Antonii 29, 1-2. 4, in G.J.M. Bartelink (ed.), Vita di Antonio [= "Vite dei Santi", 1], Fondazione Lorenzo Valla [s.l.] 1981³ , p. 66; Gb 5, 23 = Vita S. Antonii 51, 5, in Bartelink (ed.), op. cit., p. 104; Gb *40, 11 = Vita S. Antonii 5, 3, in Bartelink (ed.), op. cit., p. 16; Gb 40, 19-24 = Vita S. Antonii 24, 4, in Bartelink (ed.), op. cit., p. 56; Gb ${ }^{*} 41,10-13$ = Vita S. Antonii 24, 1, in Bartelink (ed.), op. cit., p. 54; Gb 41, 19-23 = Vita S. Antonii 24, 2-3, in Bartelink (ed.), op. cit., pp. 54 e 56.

7 Cf. Gb ${ }^{\star} 14,4=$ De baptismo 1, 2, 7, in PG 31, col. 1536 C; Gb ${ }^{\star} 34,18=$ De baptismo 2, 4, 2, in PG 31, col. 1588 C.

8 Cf. Gb 1, 4= De iudicio Dei 5, in PG 31, col. 664 C. 
volte, di cui due volte ad litteram ${ }^{9}$. Nelle Regulae fusius tractatae, invece, egli fa riferimento al Libro di Giobbe quattro volte, di cui due ad litteram ${ }^{10}$.

San Giovanni Cassiano, nelle Conlationes Patrum, adopera Giobbe addirittura ventiquattro volte, di cui fornisce sedici citazioni ad litteram $^{11}$. Nel De institutis coenobiorum, invece, egli si serve di Giobbe soltanto due volte, ma lo fa letteralmente ${ }^{12}$.

San Giovanni Crisostomo, nell'Adversus eos qui apud se habent virgines subintroductas, cita Giobbe letteralmente solamente una sola volta ${ }^{13}$. Anche nell'Adversus oppugnatores vitae monasticae, riporta Giobbe letteralmente una sola volta ${ }^{14}$. Nelle Paraeneses ad Theodorum lapsum, cita Giobbe letteralmente anche una sola volta ${ }^{15}$.

Diadoco di Fotice, nei Capita centum, utilizza il Libro di Giobbe due volte testualmente ${ }^{16}$.

9 Cf. Gb 1, 21 = Regulae brevius tractatae 262, in PG 31, col. 1260; Gb *6, $6=$ Regulae brevius tractatae 266, in PG 31, col. 1264 B; Gb ${ }^{\star} 12,11$ (LXX) = Regulae brevius tractatae 279, in PG 31, col. 1280 A.

10 Cf. Gb 2, 6 = Regulae fusius tractatae 50, 4, in PG 31, col. 1049 BC; Gb *8, $21=R e$ gulae fusius tractatae 17, 1, in PG 31, col. 961 C; Gb 29, 12 = Regulae fusius tractatae 15, 1, in PG 31, col. 952 B; Gb *38, 3 = Regulae fusius tractatae 23, in PG 31, col. 981 B.

11 Cf. Gb 1, 5, Gb 1, 20, Gb *1, 21, Gb 2, 8, Gb *2, 10, Gb 29, 15, Gb 31, 20 = Conlationes Patrum 6, 10, in SCh 42, pp. 230-231; Gb 1, 6 = Conlationes Patrum 18, 16, in SCh 64, p. 33; Gb ${ }^{\star} 1,9-10(\mathrm{LXX})=$ Conlationes Patrum 4, 6, in SCh 42, p. 171; Gb 1, 9-11 (LXX) = Conlationes Patrum 13, 14, in SCh 54, p. 170; Gb ${ }^{\star} 2,6(\mathrm{LXX})=$ Conlationes Patrum 7, 12, in SCh 42, p. 256; Gb *3, 23 (LXX) = Conlationes Patrum 6, 6, in SCh 42, p. 226; Gb *5, $7=$ Conlationes Patrum 7, 6, in SCh 42, p. 253; Gb ${ }^{\star} 5,18(\mathrm{LXX})=$ Conlationes Patrum 2, 13, in SCh 42, p. 129; Gb ${ }^{\star} 9,30-31$, = Conlationes Patrum 12, 6, in SCh 54, p. 130; Gb *10, 10-11 $(\mathrm{LXX})=$ Conlationes Patrum 8, 25, in SCh 54, p. 35; Gb ${ }^{\star} 15,14-15=$ Conlationes Patrum 6, 14, in SCh 42, p. 239; Gb ${ }^{\star} 15,15$ (LXX), Gb ${ }^{\star 2} 25,5=$ Conlationes Patrum 23, 8, in SCh 64, p. 151; Gb *38, 7 (LXX) = Conlationes Patrum 8, 7, in SCh 54, p. 15; Gb *39, 5-8 $(\mathrm{LXX})=$ Conlationes Patrum 18, 6, in SCh 64, p. 17; Gb ${ }^{\star} 40,3(\mathrm{LXX})=$ Conlationes Patrum 6, 11, in SCh 42, p. 233; Gb *40, 11 (LXX) = Conlationes Patrum 5, 4, in SCh 42, p. 191.

12 Cf. Gb $* 5,2$ = De institutis coenobiorum 5, 22, in SCh 109, p. 228; Gb *5, 23 (LXX) = De institutis coenobiorum 9, 8, in SCh 109, p. 376.

$13 \mathrm{Cf} . \mathrm{Gb}{ }^{\star 31}, 1=$ Adversus eos qui apud se habent virgines subintroductas 4 , in PG 47, col. 501.

$14 \mathrm{Cf}$. Gb ${ }^{\star} 1,5=$ Adversus oppugnatores vitae monasticae 3, 20, in PG 47, col. 384.

15 Cf. Gb ${ }^{* 9}, 25=$ Paraeneses ad Theodorum lapsum 2, 3, in PG 47, col. 313.

16 Cf. Gb ${ }^{\star} 14,17$ = Capita centum 100, in SCh 5, p. 161; Gb *31, 7 = Capita centum 56, in SCh 5, p. 117. 
Sant'Eucherio di Lione, nel De contemptu mundi, adopera il Libro di Giobbe una sola volta, e in più non letteralmente ${ }^{17}$.

Eutropio, nel De vera circumcisione, si serve del Libro di Giobbe una sola volta e non ad litteram $^{18}$.

Evagrio Pontico, nei Kephálaia gnostiká, utilizza Giobbe, non letteralmente, quattro volte ${ }^{19}$.

San Girolamo, nelle Epistulae di contenuto monastico, utilizza ben undici volte Giobbe, di cui otto volte ad litteram ${ }^{20}$. Nella Vita S. Hilarionis, invece, si serve di Giobbe una sola volta, e non testualmente ${ }^{21}$.

San Macario il Grande utilizza letteralmente Giobbe una sola volta, e lo fa negli Apophthegmata Patrum ${ }^{22}$. Nelle Homiliae spiritales, invece, se ne serve testualmente due volte ${ }^{23}$.

San Marco l'Eremita cita Giobbe una sola volta, ad litteram, nel De ieiunio ${ }^{24}$.

San Nilo di Ancira, nel De monastica exercitatione, si serve di Giobbe ben sei volte, di cui due volte ad verbum ${ }^{25}$. Nel De voluntaria paupertate lo adopera soltanto due volte, e non testualmente ${ }^{26}$, ma nelle Epistulae dirette ai monaci lo cita testualmente otto volte ${ }^{27}$.

17 Cf. Gb 14, 1. 5 = De contemptu mundi 135-136, in S. Pricoco (ed.), Eucherio di Lione. Il rigiuto del mondo [= "Biblioteca Patristica", 16], Firenze 1990, p. 64.

18 Cf. Gb 1, 21 = De vera circumcisione 11, in PL 30, col. 206 A.

19 Cf. Gb 1, 15 = Kephálaia gnostiká 2, 55, in PO [= Patrologia Orientalis, Paris 1903 ss. (ora edita a Turnhout)] 28/1, col. 83; Gb 40, 19, Gb 41, 25 = Kephálaia gnostiká 6, 36, in PO 28/1, coll. 231. 233; Gb 40, 25 = Kephálaia gnostiká 5, 37, in PO 28/1, col. 191.

20 Cf. Gb 1, 1 = Epistula 122, 3, in PL 22, col. 1045; Gb *1, 17, Gb *7, 1 = Epistula 130, 7, in PL 22, col. 1112; Gb ${ }^{\star} 1,21$ = Epistula 22, 31, 5, in CSEL 54, p. 193; Gb 2, 1-7 = Epistula 22, 4, 2, in CSEL 54, p. 148; Gb ${ }^{\star} 4,18$ e Gb ${ }^{\star} 14,4$ = Epistula 122, 3, in PL 22, coll. 1044-1045; $\mathrm{Gb} 33,3.9, \mathrm{~Gb}^{\star} 38,3, \mathrm{~Gb}{ }^{\star} 40,11=$ Epistula $22,11,2-3$, in CSEL 54, p. 158; Gb ${ }^{\star} 40,11=$ Epistula 130, 10, in PL 22, col. 1116.

21 Cf. Gb 1, 12-19 = Vita S. Hilarionis 23, in PL 23, col. 41.

22 Cf. Gb ${ }^{\star} 1,21=$ Apophthegmata Patrum 18, in PG 34, col. 249.

$23 \mathrm{Cf}$. Gb ${ }^{\star} 1,10-11$ e Gb ${ }^{\star} 2,2$ = Homiliae spiritales 26, 7, in PG 34, col. 677.

24 Cf. Gb ${ }^{\star 42,5-6}=$ De ieiunio 4, in PG 65, 1116 C.

25 Cf. Gb 1, 1-2 e Gb *1, 5 = De monastica exercitatione 23, in PG 79, col. 749; Gb 8, 11-12 = De monastica exercitatione 49, in PG 79, col. 780; Gb 31, 1-2 = De monastica exercitatione 14, in PG 79, col. 736; Gb 39, 5-8 = De monastica exercitatione 62, in PG 79, col. 793; Gb ${ }^{*} 41,4=$ De monastica exercitatione 29, in PG 79, col. 757.

26 Cf. Gb 39, 6-7 = De voluntaria paupertate 43, in PG 79, col. 1021; Gb 39, $26=$ De voluntaria paupertate 27, in PG 79, col. 1004.

27 Cf. Gb ${ }^{\star 2}, 5$ = Epistula 3, 222, in PG 79, col. 485; Gb *4, $11=$ Epistula 3, 265, in PG 79, col. 516; Gb ${ }^{\star} 7,8$ e Gb ${ }^{\star} 17,9=$ Epistula 3, 33, in PG 79, col. 393; Gb ${ }^{\star} 12,4$ e Gb 12, $5=$ 
Sant'Orsiesi, nelle Catecheses, si serve di Giobbe una sola volta e non letteralmente $^{28}$. Nella Doctrina de institutione monachorum utilizza Giobbe anche una sola volta, ma ad verbum ${ }^{29}$. Si serve di Giobbe testualmente, ma soltanto una volta, nelle Epistulae ${ }^{30}$.

San Pacomio cita Giobbe nelle Epistulae una sola volta, ma letteralmente ${ }^{31}$. Nella Vita Bohairice scripta S. Pachomii si incontra sei volte l'uso di Giobbe, di cui quattro volte ad litteram ${ }^{32}$. Nelle S. Pachomii Vitae Graece, invece, si contano persino diciassette citazioni tratte da Giobbe, di cui quattro fornite ad verbum ${ }^{33}$.

Epistula 2, 108, in PG 79, col. 248; Gb *38, 8 = Epistula 4, 45, in PG 79, col. 572; Gb *40, $3=$ Epistula 3, 321, in PG 79, 537.

28 Cf. Gb 31, 15 = Catecheses a. 5, in CSCO [= Corpus Scriptorum Christianorum Orientalium] 160, p. 74.

29 Cf. Gb ${ }^{\star} 5,14$ = Doctrina de institutione monachorum 35, in H. Bacht (ed.), Das Vermächtnis der Ursprungs [= "Studien zur Theologie des geistlichen Leben", 5], Würzburg 1972, p. 146.

30 Cf. Gb ${ }^{\star} 38,7$ = Epistula ad Theodorum, in CSCO 160, p. 66.

31 Cf. Gb ${ }^{\star} 4,11=$ Epistula 4, in PL 23, col. 97.

32 Cf. Gb ${ }^{\star} 1,21$ = Vita Bohairice scripta S. Pachomii 183, in CSCO 107, p. 105; Gb 2,7 = Vita Bohairice scripta S. Pachomii 102, in CSCO 107, p. 85; Gb * 6, $11=$ Vita Bohairice scripta S. Pachomii 192, in CSCO 107, p. 118; Gb ${ }^{\star} 16,20=$ Vita Bohairice scripta S. Pachomii 207, in CSCO 107, p. 134; Gb ${ }^{\star}$ 19, 23 = Vita Bohairice scripta S. Pachomii 194, in CSCO 107, p. 120; Gb 31, 7 = Vita Bohairice scripta S. Pachomii 197, in CSCO 107, p. 123.

33 Cf. Gb ${ }^{\star} 1,21$ = S. Pachomii Vitae Graece. Vita Prima 90, in F. Halkin (ed.) [= "Subsidia hagiographica", 19], Bruxelles 1932, p. 61; Gb 1, 21 = S. Pachomii Vitae Graece. Vita Secunda 84, in F. Halkin (ed.), op. cit., p. 262; Gb ${ }^{\star} 1,21$ = S. Pachomii Vitae Graece. Vita Tertia 140, in F. Halkin (ed.), op. cit., p. 346; Gb 2, 1-6 = S. Pachomii Vitae Graece. Vita Secunda 81, in F. Halkin (ed.), op. cit., p. 258; Gb 2, 5-7 = S. Pachomii Vitae Graece. Vita Quarta 22, in F. Halkin (ed.), op. cit., p. 424; Gb 2, $7=$ S. Pachomii Vitae Graece. Vita Prima 82, in F. Halkin (ed.), op. cit., p. 56; Gb 8, 9 = S. Pachomii Vitae Graece. Vita Prima 132, in F. Halkin (ed.), op. cit., p. 84; Gb ${ }^{\star} 9,10=$ S. Pachomii Vitae Graece. Vita Secunda 39, in F. Halkin (ed.), op. cit., p. 208; Gb 14, 7 = S. Pachomii Vitae Graece. Vita Secunda 78, in F. Halkin (ed.), op. cit., p. 255; Gb 14, 7 = S. Pachomii Vitae Graece. Vita Octava 166, in F. Halkin (ed.), op. cit., p. 320; Gb 16, 18 = S. Pachomii Vitae Graece. Vita Secunda 17, in F. Halkin (ed.), op. cit., p. 183; Gb ${ }^{\star} 16,20$ = S. Pachomii Vitae Graece. Vita Prima 148, in F. Halkin (ed.), op. cit., p. 94; Gb 16, 20 = S. Pachomii Vitae Graece. Vita Tertia 200, in F. Halkin (ed.), op. cit., p. 404; Gb 40, 24 = S. Pachomii Vitae Graece. Vita Secunda 61, in F. Halkin (ed.), op. cit., p. 231; Gb 40, 24 = S. Pachomii Vitae Graece. Vita Secunda 83, in F. Halkin (ed.), op. cit., p. 260; Gb 40, $24=$ S. Pachomii Vitae Graece. Vita Tertia 96, in F. Halkin (ed.), op. cit., p. 298; Gb 40, $24=$ S. Pachomii Vitae Graece. Vita Tertia 119, in F. Halkin (ed.), op. cit., p. 324. 
Palladio nella Historia Lausiaca adopera Giobbe quattro volte, di cui solamente una sola volta letteralmente ${ }^{34}$.

Pelagio nella Epistula ad Demetriadem adopera Giobbe sei volte, di cui cinque volte letteralmente ${ }^{35}$, e nella Epistula ad Pammachium et Oceanum de renuntiatione saeculi egli utilizza Giobbe testualmente una sola volta ${ }^{36}$.

Lo Pseudo Nilo, nell'Ad Agathiam, si serve di Giobbe ben tredici volte, di cui dieci volte ad litteram ${ }^{37}$.

Rufino di Aquileia (o di Concordia) pare appellarsi a Giobbe una sola volta (ovviamente non letteralmente) nella Historia monachorum ${ }^{38}$.

Scenute di Atripe adopera Giobbe due volte nel De iis, qui a monasterio discesserunt, di cui una sola volta lo fa letteralmente ${ }^{39}$, e nel suo Testamentum si incontra Giobbe una sola volta e non letteralmente ${ }^{40}$. Egli si serve di Giobbe due volte anche nel De vita monachorum, di cui soltanto una volta ad litteram ${ }^{41}$. Adopera ancora Giobbe, una sola volta e non letteralmente, nel De discrimine temporum ${ }^{42}$.

San Serapione di Thmuis utilizza Giobbe una volta soltanto, ma lo fa ad litteram, nelle Epistulae ad monachos Alexandrinos ${ }^{43}$.

34 Cf. Gb 2, 7 e Gb 12, 13-24 = Historia Lausiaca 12, 1-2, in G.J.M. Bartelink (ed.), Palladio. La Storia Lausiaca [= "Vite dei Santi", 2], Fondazione Lorenzo Valla, [s.l.] 1974, p. 54; Gb 6, 6 e Gb ${ }^{\star}$ 40, 3 = Historia Lausiaca 47, 14, in G.J.M. Bartelink (ed.), op. cit., p. 234.

35 Cf. Gb 1, 11 = Epistula ad Demetriadem 6, in PL 30, col. 22; Gb *1, 21 e Gb *31, $24=$ Epistula ad Demetriadem 6, in PL 30, col. 23; Gb ${ }^{\star} 2,3=$ Epistula ad Demetriadem 6, in PL 30, col. 22; Gb *27, 6 e Gb *31, 32 = Epistula ad Demetriadem 6, PL 30, col. 22.

$36 \mathrm{Cf} \mathrm{Gb} * 3,10-11$ = Epistula ad Pammachium et Oceanum 3, in PL 30, col. 248.

37 Cf. Gb 1, 1 ss. = Ad Agathiam 11, 3, in PG 79, col. 908; Gb *1, 1, Gb *29, 16-17, $\mathrm{Gb}^{\star} 31,13, \mathrm{~Gb}{ }^{\star 31}, 15, \mathrm{~Gb}{ }^{\star 31}, 16-17, \mathrm{~Gb}{ }^{\star 31}, 32=$ Ad Agathiam 10, 6, in PG 79, col. 896; Gb ${ }^{\star 2}, 9=$ Ad Agathiam 10, 6, in PG 79, col. 896; Gb 3, $10=$ Ad Agathiam 11, 11, in PG 79, col. 917. 920; Gb *4, 17 e Gb ${ }^{\star} 15,14=$ Ad Agathiam 11, 5, in PG 79, col. 909; Gb 29, $15=$ Ad Agathiam 10, 1, in PG 79, col. 888; Gb *40, $10=$ Ad Agathiam 2, 3, in PG 79, col. 820.

38 Cf. Gb 1, 1 = Historia monachorum 33, 1, in PL 21, col. 460.

39 Cf. Gb ${ }^{*}$, 27 = De iis, qui a monasterio discesserunt, in CSCO 96, p. 107; Gb 34, $19=$ De iis, qui a monasterio discesserunt, in CSCO 96, p. 75.

40 Cf. Gb 16, 10 = Testamentum, in CSCO 108, p. 125.

41 Cf. Gb ${ }^{\star} 24,25=$ De vita monachorum 7, in CSCO 108, p. 39; Gb 32-34= De vita monachorum 14, in CSCO 108, p. 54.

42 Cf. Gb 31, 1 = De discrimine temporum, in CSCO 108, p. 116.

43 Cf. Gb ${ }^{\star} 42,8$ = Epistulae ad monachos Alexandrinos 4, in PG 40, col. 929. 


\subsection{L'uso del Libro di Giobbe dai più antichi autori monastici. Sintesi}

Per quanto riguarda l'utilizzazione del Libro di Giobbe dai primissimi scrittori monastici (dei secoli IV-V), possiamo dire che, per quanto riguara la presenza dei Libri Sapienziali nella più antica letteratura monastica, Giobbe si trova al secondo posto subito dopo i Proverbi ${ }^{44}$. Il Libro di Giobbe, infatti, viene citato 153 volte, di cui 91 letteralmente. Questo libro deve la sua fortuna nella letteratura monastica delle origini, nonostante il suo stile narrativo, all'austera vita del protagonista che presagiva quella dei monaci; i dolori e lo spirito penitenziale, tipiche della personalità di Giobbe, erano modello alle privazioni della vita monastica; e la sua vittoria come la ricompensa finale suonavano da incoraggiamento. L'interrogativo di fondo degli asceti e dei monaci - sul perché l'uomo giusto, ossia il monaco, debba essere oppresso da tante tribolazioni - trovava in Giobbe una risposta che, cioè, esse sono soltanto prove destinate a purificare e perfezionare. La sua storia, inoltre, narrando della lotta di Satana contro il giusto e della vittoria di quest'ultimo, offriva ai monaci un solido baluardo contro le forze nemiche dei demoni. La demonologia è presente in modo quasi ossessivo nell'antico monachesimo, pervaso dal senso dell'indefesso combattimento contro il diavolo e contro i suoi alleati - l'ispirazione primordiale della vita eremitica era proprio la lotta contro il maligno, condotta nel deserto, il luogo della sua dimora, e trovava, dunque, ampia ispirazione dalla narrazione di Giobbe.

44 Cf. B. Degórski, I Libri sapienziali nella letteratura monastica delle origini (secc. IV$V$ ), in Letture cristiane dei Libri Sapienziali. XX Incontro di studiosi dell'antichità cristiana, Roma, 9-11 maggio 1991, Istituto Patristico «Augustinianum» [= "Studia Ephemeridis «Augustinianum»", 37], Roma 1992, p. 81; Idem, L’elenco delle citazioni dei Libri sapienziali nella letteratura monastica del IV e V secolo, «Roczniki Teologiczne» 40 (1993), pp. 119-154. 
I passi di Giobbe a maggior incidenza negli scritti monastici dei secoli IV-V sono i seguenti: Gb 1, 21 (dodici volte) ${ }^{45}$, Gb 2, 7 (sei volte) ${ }^{46}$, Gb 40, 11 (sei volte $)^{47}$, Gb 40, 24 (cinque volte) ${ }^{48}$.

Esisteva un commento a tutto il libro di Giobbe, scritto da Evagrio Pontico, ma esso è andato perduto; M. Faulhaber ne ha trovati venticinque scholia nel codice manoscritto Vallicellanus C. $41^{49}$, conservato nella "Biblioteca Vallicelliana", a Roma.

Scorrendo diffusamente le citazioni dei libri sapienziali come tali e, in seno ad essi, del Libro di Giobbe, saltano allocchio due modi particolari nei quali esse vengono inserite nel contesto:

$1^{\circ}$ ) come facenti parte del discorso stesso dell'abbas, tralasciandone la provenienza biblica, e senza alcuna spiegazione precedente o successiva che ne esprima il medesimo concetto in termini personali dell'autore ${ }^{50}$. È, questo, un modo di citare la Sacra Scrittura molto diffuso nella letteratura ascetica in ge-

45 Cf. Apophthegmata Patrum. Macarius Aegyptius 18, in PG 65, col. 269; Iohannes Cassianus, Conlationes Patrum 6, 10, in SCh 42, p. 231; Hieronymus, Epistula 22, 31, 5, in CSEL 54, p. 193; Macarius Aegyptius Magnus, Apophthegmata 18, in PG 34, col. 249; Pachomius, Vita Bohairice scripta 183, in CSCO 107, p. 105; Idem, Vita Prima 90, in F. Halkin (ed.) [= "Subsidia hagiographica", 19], Bruxelles 1932, p. 61; Idem, Vita Tertia 140, in F. Halkin (ed.), op. cit., p. 346; Pelagius, Epistula ad Demetriadem 6, in PL 30, col. 23; e non testualmente da Basilius Caesariensis, Regulae brevius tractatae 262, in PG 31, col. 1260; Iohannes Cassianus, Conlationes Patrum 6, 10, in SCh 42, p. 230; Pachomius, Vita Secunda 84, in F. Halkin (ed.), op cit., p. 262.

46 Per le citazioni non letterali cf. Athanasius Alexandrinus, Vita S. Antonii 1-2. 4, in G.J.M. Bartelink (ed.), op. cit., p. 66; Hieronymus, Epistula 22, 4, 2, in CSEL 54, p. 148; Pachomius, Vita Bohairice scripta 102, in CSCO 107, p. 85; Idem, Vita Quarta 22, in F. Halkin (ed.), op. cit., p. 424; Idem, Vita Prima 82, in F. Halkin (ed.), op. cit., p. 56; Palladius, Historia Lausiaca 12, 1-2, in G.J.M. Bartelink (ed.), Palladio. La Storia Lausiaca [= "Vite dei Santi", 2], Fondazione Lorenzo Valla, [s. 1.] 1974, p. 54.

47 Cf. Athanasius Alexandrinus, Vita S. Antonii 5, 3, in G.J.M. Bartelink (ed.), op. cit., p. 16; Iohannes Cassianus, Conlationes Patrum 5, 4, in SCh 42, p. 191; Hieronymus, Epistula 130, 10, in PL 22, col. 1116; ibidem 130, 10, in PL 22, col. 1116; e non letteralmente da Antonius Abbas, Epistula 17, in PG 40, col. 1044 B; Idem, Sermo 20, in PG 40, col. 976.

48 Per le citazioni non ad litteram, cf. Athanasius Alexandrinus, Vita S. Antonii 24, 4, in G.J.M. Bartelink (ed.), op. cit., p. 56; Pachomius, Vita Secunda 61, in F. Halkin (ed.), op. cit., p. 231; ibidem 83, in F. Halkin (ed.), op. cit., p. 260; Idem, Vita Tertia 96, in F. Halkin (ed.), op. cit., p. 298; ibidem 119, in F. Halkin (ed.), op. cit., p. 324.

49 Cf. M. Faulhaber, Proverbien und Predigerkatenen, Wien 1902.

50 Cf. come esempio: Augustinus Hipponensis, De opere monachorum 28, 36, in CSEL 41, p. 585: "[...] ne dicant animae bonae: «post odorem unguentorum tuorum curremus», et sic laqueos evadant [...]". 
nere, che non rielabora concettualmente il dato biblico, ma lo incorpora tout court nel discorso; le citazioni, infatti, sono implicite e introdotte per asindeto;

$2^{\circ}$ ) come appoggio all'insegnamento appena proposto dall'abbas, il quale insegnamento, evidentemente, scaturisce dalla meditazione dei testi biblici, ma viene riformulato dall'autore e, talvolta, comporta un tentativo interpretativo. In questi casi, l'interpretazione non è personale, ma rifluisce nell'interpretazione comunemente circolante negli ambienti monastici, frutto, probabilmente, di contatti espliciti e diretti fra gli $a b b a$ (che, sappiamo, condividevano e confrontavano reciprocamente gli insegnamenti spirituali ${ }^{51}$ ) e, comunque, testimone di una tradizione monastica vivente. In questo secondo caso di larvata esegesi, contrariamente al primo, le citazioni sono precedute da formule (o simili): sicut scriptum est, dicitur [...], che ne evidenziano il carattere esemplificativo.

Esiste un unico caso di spiegazione del testo biblico in se stesso, cioè di esegesi in senso proprio: le Epistulae di san Nilo di Ancira, che mirano a spiegare la littera del Cantico $^{52}$. Esse restano, però, un esempio isolato e appena germinale.

\section{Rilievi conclusivi}

La letteratura monastica delle origini presenta un impiego relativamente alto dei libri sapienziali come tali, presi nel loro insieme e senza marcare la differenza fra letteratura orientale ed occidentale e in seno ai singoli autori. Il fatto non è sorprendente se si guarda al carattere insieme spirituale e formativo, nonché alla comunanza della regola di vita che rendevano questi testi particolarmente idonei alla sensibilità e alla crescita ascetica dei primissimi monaci.

Laddove lo stile sia sentenzioso, non soltanto il Libro di Giobbe, ma anche tutti gli altri libri sapienziali diventano addirittura ispiratori dello stile a massima degli insegnamenti degli $a b b a$, e possono essere interpretati come elementi integranti i discorsi ai monaci.

Stile sapienziale e stile monastico costituiscono una combinazione singolare, peculiare e felice, lontana da una valenza esegetica in senso stretto e da una attenzione dottrinale, ma non per questo priva di pregnanza e di importanza ai fini di una tradizione interpretativa vivente, che configura i libri sapienziali come tali e, in seno ad essi, il Libro di Giobbe, come rivelazione di contenuto ascetico e gli scritti monastici come letteratura a carattere "sapienziale".

51 Gli Apophthegmata Patrum, ad esempio, ampiamente testimoniano dei contatti fra i Padri del deserto.

52 Cf. Nilus Ancyranus, Epistulae 1, 333, in PG 79, col. 201. 
